\title{
REVIVIENDO A SHAKESPEARE: RETOS Y MONTAJES
}

\author{
Remedios Perni Llorente, Universidad de Murcia ${ }^{1}$ \\ Email: rperni@um.es
}

Resumen: Cuando, en 1968, Roland Barthes anunció la muerte del Autor, muchos críticos propusieron liberar la crítica de la tiranía autoral y sus inescrutables intenciones. Sin embargo, Shakespeare -o por lo menos su halo espectral- se mantiene omnipresente en la cultura occidental. La cultura visual -la pintura, el cine, los pósters- mantiene "viva" la imagen de Shakespeare, mientras que la idea de "autenticidad" continúa siendo la causa de acalorados debates. ¿Qué queremos decir hoy día cuando nombramos a Shakespeare? ¿Es posible revivirlo? Cierta creencia nostálgica todavía mantiene que cualquier Shakespeare pasado fue mejor, mientras que otras perspectivas apoyan la posibilidad de que Shakespeare se recicle con el tiempo.

Palabras clave: Shakespeare, autoría, artes visuales, cultura visual, teoría crítica

Título en inglés: "Reviving Shakespeare: remains and montages"

Abstract: When, in 1968, Roland Barthes announced the death of the Author, many critics proposed freeing critical practice from the authorial tyranny and its inscrutable intentions. Nonetheless, Shakespeare -or at least his ghostly halo- maintains an all-pervading presence in Western Culture. Visual culture -paintings, films, posters- keeps Shakespeare's image "alive," while the idea of "authenticity" continues being the source of over-heated debates. What do we mean today when we name William Shakespeare? Is it possible to revive Shakespeare? Certain nostalgic belief still stands that any Shakespeare past was better, whereas other perspectives support the ability to recycle the Shakespearean image over time.

Keywords: Shakespeare, authorship, visual arts, visual culture, critical theory

Is Shakespeare Dead?

Mark Twain

La búsqueda de un retrato auténtico de Shakespeare ha provocado diversas frustraciones desde que en 1769 David Garrick propulsó el Jubilee, celebración del nacimiento del poeta en Stratford-upon-Avon. Esta gran fiesta de conmemoración estableció dicho lugar

1 Date of reception: 1 August 2015

Date of acceptance: 9 November 2015 
como punto neurálgico y de peregrinaje para los shakesperianos, al tiempo que propulsó el ascenso del bardo a una suerte de Olimpo de autores que aún hoy tiene sus devotos (Bate 2003: 19). El propósito del presente texto es ofrecer un recorrido por las ansiedades generadas en torno al rostro de Shakespeare una vez se comenzó a promover la bardolatría y la repercusión que el ánimo de revivirlo y/o recrearlo ha tenido en el legado visual shakesperiano de las últimas décadas.

Así pues, este recorrido se inicia en el siglo XVIII, pero no puede comprenderse en toda su amplitud sin aludir a las nociones sobre genialidad que proliferaron en el siglo XIX y sus vinculaciones con la obra shakesperiana. Por entonces, basándose en la noción de que el espíritu del genio emana infinitamente del texto -incluso después de la muerte física- la mitología romántica definió la manera de rendir tributo al autor consagrado. El autor vino a encarnar el mito de "los dos cuerpos del rey", estando en disposición tanto de "el cuerpo terrestre", sujeto a los estragos del tiempo, como del "cuerpo sublime, inmaterial y sagrado", invulnerable a éste (Lehmann 2002: 2). Así, podría decirse que para muchos de sus lectores, Shakespeare -en tanto que autor sublime, inmaterial y sagrado- y su obra conformaban (y aún conforman) un solo cuerpo. Tal vez por eso, en numerosas ocasiones, el nombre del autor se utiliza de forma indistinta para referirse a la obra textual y al 'genio' responsable de ella. Este ser amalgamado, obra y autor firmante, sigue formando parte del imaginario académico y artístico.

Recordemos, por un lado, las disquisiciones de críticos como Gary Taylor, para quien el Autor Shakespeare es la causa primera de la obra, por reunir ésta las condiciones de calidad y estilo que caracterizan al poeta. ${ }^{2}$ Hallamos ideas afines al ensalzamiento autoral de Shakespeare en numerosos monográficos; por mencionar algunos: Shakespeare, The World as Stage (Byson 2007), Shakespeare, a Life in Drama (Wells 1995), Shakespeare for all Time (Wells 2003), A Year in the Life of William Shakespeare: 1599 (Shapiro 2005), o Soul of the Age: A Biography of the Mind of William Shakespeare (Bate 2010). Por otro lado, películas como Shakespeare in Love (John Madden, 1998), y su éxito en taquilla, atestiguan la pervivencia de la figura romántica del genio bohemio y su buena acogida por parte del público en las últimas décadas. Como observa Mark Twain, en su ensayo Is Shakespeare Dead?, no ha existido personaje histórico con existencia tan escasamente documentada que haya generado mayor impacto en la memoria colectiva. En efecto, se da la paradoja de que, a pesar de que no existe certidumbre sobre la apariencia de Shakespeare, todos reconocemos de inmediato su imagen.

Asimismo, ocurre que esta actitud, proclive a la personificación y devoción al genio, ha suscitado posicionamientos contrarios en las últimas décadas del siglo XX y hoy día. Cuando en 1968, Roland Barthes, anunció la muerte de esos dos cuerpos autorales, críticos del extremo opuesto se propusieron liberar la práctica crítica de la tiranía del autor y sus insondables intenciones. Así, durante los últimos veinte años, no sólo se ha declarado muerto a Shakespeare, también ha sido desmembrado: in-nombrado por Margreta De Grazia,

2 Gary Taylor ha reiterado la primacía de la autoría en estudios literarios, dedicándose, en la actualidad, a reivindicar a Thomas Middleton como "el otro Shakespeare." Así lo hizo en su conferencia plenaria para la Britgrad Conference de 2008, que comenzó cuestionando la validez de las ideas expuestas por Michel Foucault en “Qu'est-ce qu'un auteur?” (1969). 
des-puntado por Michael Warren, des-corregido por Randall McLeod y des-editado por Leah Marcus (Lehmann 2002: 5).

Sin embargo, a pesar de las conclusiones casi nihilistas a las que algunos críticos han llegado a partir del ensayo de Barthes o el de Michel Foucault, Qué es un autor (1969), el fenómeno Shakespeare va más allá de lo que Margreta de Grazia o Peter Stallybrass considerarían "un efecto del texto" (de Grazia y Stallybrass 1993: 9). Aún cuando éstos intentan centrar su análisis en aspectos puramente materiales, caen de forma paradójica en una suerte de "tratamiento antropomórfico del texto" (Lehmann: 2002: 14). Apuntan, por ejemplo: "in the sheets of a book, bedsheets began a new life", implicando una transferencia nada sutil de placer físico entre las sábanas de una cama al 'placer textual' entre las páginas de un libro. También cuando mencionan la aparición de cuerpos extraños en esas páginas ("rags, juniper gum" o "the residual traces of the urine of the printshop workers"), contenidos todos ellos en la absorbente superficie del texto shakesperiano, parecen querer dar a entender que cualquier cuerpo, menos el autoral, está invitado a participar en esa escena primigenia de "placer textual." Y, sin embargo, pese a la insistencia en estos aspectos, no pueden llegar a deshacerse de la perenne presencia de Shakespeare, al que aluden como "cosa fantasmagórica" (ghostly thing) en la conclusión de su ensayo, sugiriendo por tanto que hay en el texto algo más que el texto (2002: 18).

Por una parte, los textos dramáticos del renacimiento inglés eran radicalmente inestables debido a las variaciones a las que se les sometía para su puesta en escena. Sabemos que servían como guiones que luego podían ser manipulados por los actores o por otros dramaturgos colaboradores y las representaciones solían durar menos de lo previsto por el texto completo: "the text, then, was not the play" (Orgel 2002: 21). Teniendo en cuenta este contexto de producción, no puede considerarse a William Shakespeare el único responsable del material dramático que nos ha legado. Por otra parte, Shakespeare -en tanto que autor- resulta ineludible en el análisis de su obra en cuanto "algo más allá." Orgel observa:

What is authentic here is something that is not in the text; it is something behind it and beyond it that the text is presumed to represent: the real life of the characters, the actual history of which the action is a part, the playwright's imagination, or the hand of the master, the authentic witness of Shakespeare's own history. The assumption is that texts are representations or embodiments of something else and that it is that something else which the performer o editor undertakes to reveal. What we want is not he authentic play, with its unstable revisable script, but an authentic Shakespeare, to whom every generation's version of a classic drama may be ascribed (2002: 256).

No obstante, aún optando por reconocer que la sombra de Shakespeare, su espectral halo, se alarga de alguna manera sobre su obra dramática, el enigma sigue sin resolverse, no encuentra concreción. ¿A qué nos referimos, todavía hoy, cuando nombramos a William Shakespeare? Esta incertidumbre ha supuesto una fuente de disensiones y una mina inagotable de matices contrapuestos y complementarios, sobre todo a la hora de decidir a qué elementos del fenómeno se le debería otorgar mayor énfasis. Para unos, la tarea a desempeñar en el estudio de Shakespeare debe centrarse en su revival: buscarle para devolver la vida a la lectura original; el hallazgo y la resurrección del sagrado rostro. Para otros, en cambio se trataría más bien de llevar a cabo un reciclaje, de recuperar los restos y realizar un montaje con ellos (Cartelli y Rowe 2007). En este sentido, 'reciclaje' y 'mon- 
taje' vendrían a confluir; y nótese que la idea de montaje conlleva connotaciones cinematográficas idóneas para trabajos sobre adaptación como el de Courtney Lehmann (2002). Curiosamente, esta visión tiene su origen en el campo de la Historia del Arte, en concreto en la idea de supervivencia (Nachleben) de Aby Warburg, y en la teoría historiográfica de Walter Benjamin, quien aborda la idea de la Historia como montaje. Lo novedoso de la idea de resto o supervivencia (lo que Warburg llama Nachleben) es que asume un modelo temporal completamente distinto al que todavía muchos académicos presuponen al estudio del Renacimiento, campo que, por denominación, se ha venido asociando con la idea de revival, renacer, revivir (Warburg 1999).

Antes de proseguir con estas teorías y a fin de ahondar en el tema que nos ocupa más adelante, detengámonos por un instante en las consecuencias que la bardolatría impulsada por David Garrick tuvo sobre el medio visual. Éstas pueden intuirse en el problema que Thomas Gainsborough expone en la siguiente carta, dirigida al actor en 1769:

I have been several days rubbing in and rubbing out my design of Shakespeare, and hang me, if I think I shall let it go, or let you see it at last. I was willing like an ass as I am, to expose myself a little out of the simple portrait way, and had a notion of showing where that inimitable poet had his ideas from, by an immediate ray darting down upon his eye turned up for the purpose; but, confound it, I can make nothing of my ideas -there has been such a fall of rain from the same quarter. You shall not see it, for I will cut it before you can come. Tell me, dear sir, when you purpose coming to Bath, that I may be quick enough in my motions. Shakespeare's bust is a silly, smiling thing; and I have not sense enough to make him more sensible in the picture, and so I tell ye, you shall not see it. I must make a plain picture of him standing erect, and give it an old look, as if it had been painted at the time he lived; and there we shall fling'em. I am, dear sir, your most obedient, humble servant. (Hartman 1901: 12)

Previo a esta carta, David Garrick se había puesto en contacto con Thomas Gainsborough para que rehiciera un cuadro que había pintado en 1766 en el que aparecía el propio Garrick apoyado en un pedestal sobre el que se erguía un busto de Shakespeare. El trabajo de Thomas Gainsborough, como el de William Hogarth, podía contribuir a ensalzar no sólo las fiestas en honor a Shakespeare, sino su propia imagen; como sabemos, Garrick llegó a proclamarse descendiente del bardo (Baugh 2003: 29). Sin embargo, como se hace patente en la carta de Gainsborough, llevar a cabo un nuevo retrato no resultaba una tarea sencilla. Seguramente no fue la lluvia torrencial que cayó por aquellos días sobre Stratford-upon-Avon lo que disuadió al pintor de acercarse a la fiesta con un cuadro recién pintado; temía, sobre todo, presentarse con un trabajo que no estuviera a la altura de Shakespeare. En su carta, Gainsborough se queja de que el busto que tiene ante sí -y que no llega a mejorar- es "una cosa tonta y sonriente" y que tal vez debiera llevar a cabo una obra distinta, con Shakespeare de pie y erguido y con aspecto antiguo, como si el retrato hubiera sido pintado en los tiempos en los que vivió. ${ }^{3}$

No se conocen con exactitud los acontecimientos que siguieron a esta carta. De acuerdo con Sadakichi Hartman, que investigó este asunto hacia 1900, Gainsborough había enviado

3 Gainsborough se refiere, probablemente, al busto que se encuentra en la Trinity Church de Stratford-upon-Avon, junto a la tumba de Shakespeare. 
otra misiva anunciando que "Shakespeare llegaría sin dilación", pero parece que el retrato nunca fue terminado ni visto por nadie aparte del artista (Hartman 1901: 12). Posteriormente, otros investigadores han ubicado la obra en el Town Hall de Stratford-upon-Avon hasta 1946, año en el que la destruyó un incendio (Wells 2003: 224). Existe un grabado, realizado por Valentine Green, que reproduce la imagen de Garrick y el busto de Shakespeare realizada por Gainsborough, pero sigue siendo un misterio si aquel "Shakespeare de pie y erguido", al que se refiere el pintor en esa carta tan llena de turbación, llegó a adquirir forma. En cualquier caso, Hartman opina que:

It may be safely said that the world is not much the loser by this failure, and that Shakespearean scholars do not need to regret it overmuch. Undoubtedly it would have been interesting, for few Englishmen have handled the brush with as much elegance and dexterity as Gainsborough, but, after all, it would have remained an ideal portrait, an attempt at a posthumous likeness, which in no way could have solved the mystery of Shakespeare's appearance (Hartman 1901: 13).

Lo más destacable de esta cuestión no reside ya tanto en averiguar el paradero del retrato de Gainsborough como en reflexionar sobre los quebraderos de cabeza que provocó en éste. Según nos cuenta, delinear los contornos faciales de Shakespeare requería inspiración y respeto; no bastaba con un busto "tonto y sonriente", era fundamental rescatar esa apariencia de antaño, la que tuviera Shakespeare en sus tiempos; la pintura debía invocar el pasado ante los ojos de sus espectadores. Al pintor le avergonzaba fracasar en tal empresa. Hartman, siglos después, no se quedó rezagado en esta disposición de adoración al bardo. Para él, de no haberse ejecutado nunca la idea de Gainsborough, no nos habríamos perdido gran cosa ya que, después de todo, su afán respondía a la fantasía de recobrar un parecido póstumo que mantendría irresoluto el "misterio" de la apariencia de Shakespeare. Para Hartman, los pinceles de Gainsborough nunca habrían sido capaces de evocar al genio que oculta su rostro tras los velos del tiempo.

Aunque a primera vista pudieran resultar distintas, ambas posturas se yerguen sobre un mismo principio, el culto a Shakespeare. Es posible intuir en las palabras de Hartman, a pesar de todo, cierta idealización. Utilizando el término específico atribuido a este fenómeno desde la conmemoración de Stratford-upon-Avon, su descontento viene determinado por los efectos de la bardolatría, le entristece no poder recrear el rostro perdido, no ser capaz de recuperar el pasado. La nostalgia impide a Gainsborough y Hartman aceptar un retrato libre y novedoso de un poeta al que quisieran conservar tal cual en su memoria y en la memoria de los demás. La de Shakespeare es una fuerza que ha de reproducirse tal y como debió ser. De lo contrario, sólo restan dos opciones: reconocer que cualquier intento por conseguirlo es vano o, siendo optimistas, creer que el tiempo devolverá la dicha. Que Shakespeare volverá o que está por venir. Tal actitud sigue ejerciendo una poderosa influencia en nuestros días, cuando Shakespeare continúa siendo "el autor más leído del mundo" (Jackson 2007: 18), el inventor de "lo humano" (Bloom, 1999), "el esperanto cultural" (Howard y O'Connor 2004: 13) e incluso "una necesidad religiosa" (Cartmell 200: 3). Shakespeare está tan imbuido en nuestra cultura que inspira devociones religiosas, por lo que no es de extrañar que bajo el sacro influjo del bardo, Wilson Knight escribiera durante las turbulencias de la Segunda Guerra Mundial: "We need expect no Messiah, but we might, at this tour, turn 
to Shakespeare, a national prophet if ever there was one, concerned deeply with the royal soul of England" (Knight, 2002).

La incertidumbre de Gainsborough, por tanto, viene dada por la imposibilidad de salvar la distancia entre el venerado rostro 'original' y su retrato. Pero, ¿hasta qué punto un retrato -o una imagen en general- puede reproducir con exactitud un concepto como Shakespeare, el autor de dos cuerpos, la amalgama de cuerpo y texto, el poeta de existencia ambigua, improbable y fantasmagórica?

Jean Clair, en su conocido ensayo Trazo por trazo, diente por diente (1999), subraya que la fidelidad mimética de la imagen a su modelo tiene "el carácter lábil de no tener que designar su objeto", siendo el retrato "aquello que resbala, que circula, vector de una autoridad que no remitiría a la descripción de un individuo sino a la autoridad de un significante que avala lo que dice hasta el punto de no necesitar ningún referente para existir." Es decir, "un retrato se impone en cuanto retrato porque insiste en su significancia de retrato y no porque remite a una representación, que sería por ejemplo la faz de Cristo" (Clair 1999: 161).

El retrato señala el lugar de lo simbólico. Cuando en un fresco, un retablo, una moldura de arquitectura, pasma con su poder nuestra mirada es porque, en medio de una teoría de rostros indiferentes o anónimos, aparece bruscamente con una evidencia análoga a la convicción: ese retrato, entre todos los demás, es el retrato de un hombre que existió y que no se parecía a ningún otro (1999: 161-162).

Jean Clair, añade que "la virtud del retrato reside en que jamás podría ser tomado al pie de la letra" (162). El retrato no es el retratado, sino que ocupa su lugar, el lugar de lo que falta. Así funcionan las imágenes del arte. Retratos tomados al pie de la letra, serían el de Narciso reflejándose en el agua o el de Dorian Grey en el espejo, cuya imagen se nutre de la sangre de su víctima. También las que produjeran por adherencia a la cara del fallecido aquellos que querían conservar el rostro de sus ancestros, poniendo en contacto directo la materia (el yeso) con la materia (el rostro). ${ }^{4}$ Podríamos aludir incluso a las supuestas facciones de Cristo en la Sábana Santa. Es decir, el retrato susceptible de ser tomado al pie de la letra es aquel que se piense vinculado directa y materialmente con el retratado, no consistente entonces en una imitación sino en una suerte de soporte de su existencia y de su memoria.

Thomas Gainsborough podría haber saciado su insatisfacción aproximándose a la apariencia de "los tiempos de Shakespeare", pero para historiadores como Hartman, sus intentos responden a un idealismo desdeñable. Sus trazos no pueden tomarse al pie de la letra. El gran misterio de la faz de Shakespeare permanece irresoluto. Por otra parte, esa faz hubo de existir y podría reaparecer plasmada en alguna tela, parecen pensar Gainsborough, Hartman y muchos otros shakesperianos. Y si existe, hay que buscarla. Ir por el buen camino. Serle fiel a una idea. ${ }^{5}$

4 Tal y como Plinio el Viejo cuenta en el libro XIV de su Historia Natural.

5 Se ha especulado incluso sobre la existencia de uno de esos retratos al pie de la letra, en concreto sobre cierta mascarilla funeraria realizada en 1616, descubierta por Hildegard Hammerschmidt-Hummel en 2006 y que hoy día se encuentra en Darmstad (Alemania), pero no existe evidencia definitiva de que se trate de una reproducción de la cara de Shakespeare. (Véase la noticia de dicho descubrimiento, por ejemplo, en $B B C$ News on-line: http://news.bbc.co.uk/2/hi/entertainment/4742716.stm) 
Se piensa que el grabado de Martin Droeshout, que abre la edición Folio de 1623, refleja con autenticidad al poeta, pero, para muchos bardólatras no es suficiente esta cara caricaturesca publicada siete años después de la muerte de Shakespeare. Se busca un cuadro al óleo -el óleo es un material digno de Shakespeare, una pintura que ponga de manifiesto la grandiosidad del genio, que muestre el color de su pelo y los detalles de su vestimenta con máximo mimetismo y espectacularidad.

Stanley Wells protagonizó un conocido capítulos a este respecto al anunciar al mundo su descubrimiento del retrato definitivo de William Shakespeare: una pintura conservada por la familia irlandesa Cobbe, de la que no se había tenido noticia hasta 2008. Fechada en 1610, podría haber servido de modelo para el retrato de Martin Droeshout. ${ }^{6}$ Wells ha argumentado el parecido entre ambos como prueba concluyente.

Sin embargo, existen numerosos casos de descubrimiento fallido, muchos investigadores se han precipitado a anunciar que ha encontrado a Shakespeare con pruebas poco contundentes. En el año 2005, por ejemplo, una prueba de rayos x desmintió la fecha inscrita en el Flower Portrait, un retrato que fue datado en 1606 pero que resultó haber sido pintado en el siglo XIX. En el año 2006, la National Gallery de Londres organizó la exposición titulada Searching Shakespeare, donde se daba cierto protagonismo al retrato Chandos, obra de John Taylor perteneciente al Duque de Chandos. Sin embargo, el parecido que guarda con el Droeshout o con el busto escultórico que se conserva en la Holy Trinity Church de Stratford-upon-Avon, que también se considera auténtico (aunque poco 'realista' para lo que le gustaría al público shakesperiano), es nulo. Más decepcionante, si cabe, es el caso del retrato Sanders, ya que después de que Stephanie Nolen lo mostrara al mundo en 2001, se descubrió que la fecha de ejecución no coincidía con un Shakespeare en plena juventud como el del cuadro.

En cuanto al retrato Cobbe, Katherine Duncan-Jones ha apuntado que su similitud con el Droeshout no es tan evidente, siendo en cambio muy parecido a un retrato de Sir Thomas Overbury (1518-1613) que se conserva en la Bodleian Library desde 1740. Además, tal y como indica su indumentaria, Sir Thomas Overbury perteneció a la nobleza, como el hombre del retrato Cobbe, mientras que Shakespeare nunca disfrutó de ese rango:

Though a leading 'King`s Man', Shakespeare was no nobleman, and even his status as 'gentleman' was repeatedly called in question by some of the heralds. (As John Davies of Hereford records, both Shakespeare and Burbage hoped for further preferment from James I, but didn't get it.) When players dressed above their rank offstage, it tended to get them into trouble. It is hard to believe that Shakespeare would have been rash enough to permit himself to be portrayed in such a grand array (Duncan-Jones 2009: 7).

6 Véase el testimonio de Stanley Wells realizado para la web de The Shakespeare Birthplace Trust, donde se ha expuesto este retrato acompañado del título "Shakespeare Found": http://www.shakespearefound.org.uk/ evidence.html. Último acceso: Agosto 2015. 


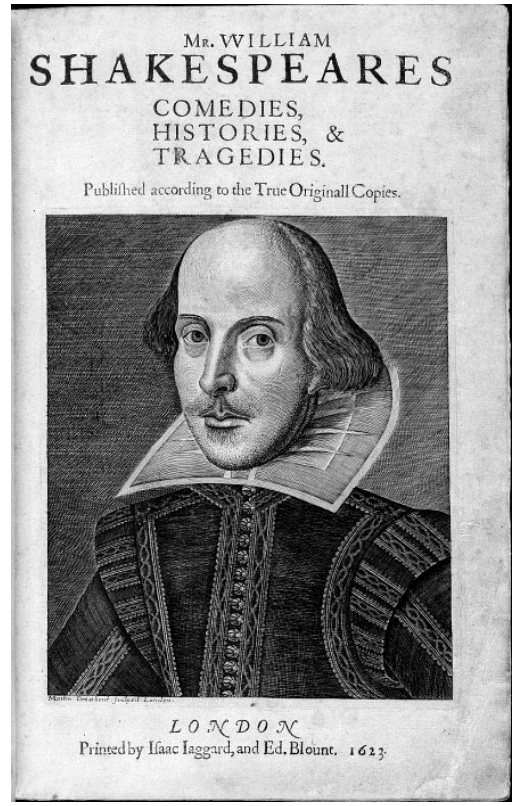

Folio Portrait. Martin Droeshout. 1623. Fuente: Wikimedia.

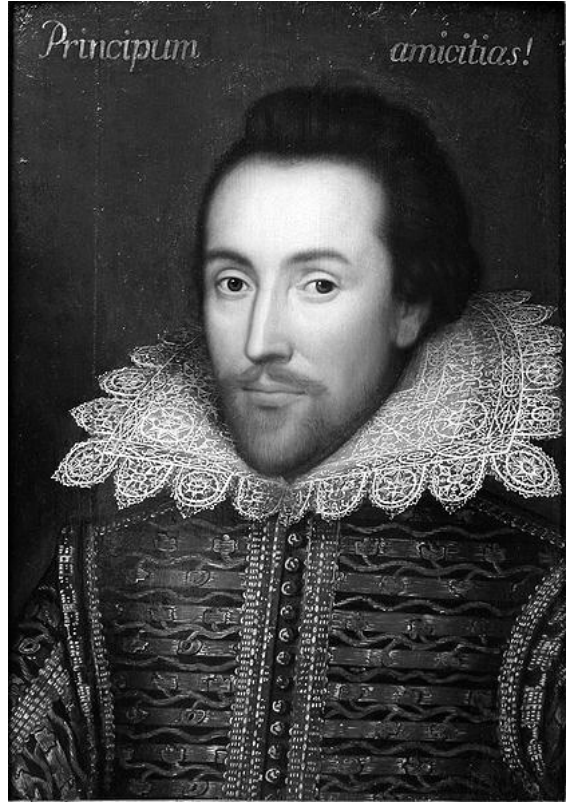

Cobbe Portrait. Artista desconocido. 1610. Fuente: Wikimedia. Getty Images.

Las razones esgrimidas por Stanley Wells no sólo obvian este hecho, sino que encuentran en él su apoyo fundamental. De acuerdo con Wells, lo novedoso del Retrato Cobbe es precisamente que descubre al público de Shakespeare "a man who was of high social status" (citado por Lacayo, Time 2009). Wells afirma no albergar dudas de que se trate del original, pues, en comparación con el Retrato de Sir Thomas Overbury, el de Shakespeare es "a much livelier painting, a much more alert face, a more intelligent and sympathetic face” (Lacayo 2009). A estas apreciaciones podemos sumar las de la institución que ha acogido la exposición del retrato, la Shakespeare Birthplace Trust; en la web donde anuncian el programa de la exposición, incluye unas palabras de Henry Woudhuysen quien apunta que Sir Thomas Overbury, "to our mind was not quite as good looking as Shakespeare" (Woudhuysen, 2009).

¿Es lícito justificar la autenticidad de un retrato de Shakespeare, parecido al grabado de 1623 , porque el retratado sea un hombre de alto rango y aire inteligente? En primer lugar, es indemostrable que Shakespeare fuera un aristócrata y, hasta el momento, los pocos resquicios fehacientes parecen apuntar a que nunca fue así. Si, como apunta Wells, este cuadro demuestra que Shakespeare disfrutó de un alto estatus socioeconómico, ¿qué es lo que, a su vez, demuestra que el cuadro es auténtico, aparte de su fecha de ejecución, si el parecido con el grabado Droeshout también puede ser puesto en entredicho? Wells parece apelar al sentir fanático de los shakesperianos al subrayar el gesto despierto, inteligente y 
empático del retrato. En el catálogo de la exposición Shakespeare Found (2009) incluso se apunta que Sir Thomas Overbury no era tan atractivo como Shakespeare.

Los argumentos de Wells denotan no sólo el lógico interés de algunos historiadores por descubrir el verdadero pasado de Shakespeare sino también una fascinación idealizadora (Shakespeare era más rico y más atractivo de lo que habíamos pensado hasta el momento). Esta idealización nos conduce de nuevo al caso de Gainsborough y a los comentarios de Hartman. La cuestión de la recuperabilidad del pasado de Shakespeare sigue en pie. No hay elogios suficientes para sublimarlo en el presente y se mantiene en pie la creencia nostálgica de que cualquier Shakespeare pasado fue mejor. Dar con él, hacerlo volver, revivirlo, se convierte entonces en una tarea frustrante, cuando no en una fuente de espejismos.

¿Cómo analizar este fenómeno de una forma satisfactoria en el seno de la academia? Thomas Cartelli y Katherine Rowe lo hacen al contrastar la noción de revival con la de recyling; la primera se refiere a la mencionada ansia revitalizadora de Shakespeare; la segunda hace énfasis en la capacidad de reciclaje de la imagen shakesperiana a lo largo del tiempo, dejando a un lado el anhelo de restauración de un retrato único y fiel (en el sentido más tradicional de la palabra), ambas guardan una estrecha relación con la idea de montaje anteriormente mencionada. El reciclaje hace hincapié en las nuevas miradas provistas por el desarrollo tecnológico y los cambios habidos en las condiciones de producción del material cultural, permitiéndose referencias autoconscientes, citas, anacronismos y guiños irónicos, poniendo especial atención en los mecanismos de apropiación y proyectando la imagen reciclada a un nivel extra-Shakesperiano (2007: 6). Encontramos también respuestas en los trabajos de Bryan Reynolds y William N. West, quienes se refieren a este 'extra' con la expresión 'Shakespeare +' en los ensayos de Rematerializing Shakespeare (2005). Este símbolo '+' se refiere al hecho de que esos restos reconocibles de Shakespeare que nuestra cultura conserva y manipula adquieren nuevos matices en nuevos contextos.

En el ámbito del retrato, tal vez uno de los ejemplos más llamativos de rematerialización sea el póster que han utilizado la Folger Shakespeare Library, el World Shakespeare Congress y la Shakespeare Association of America para anunciar sus actividades en distintas fechas de la primera década del siglo XXI. En él vemos a Shakespeare Dude, obra de Kevin Chadwick (circa 2005). Se trata de un Shakespeare rejuvenecido, de aire socarrón, bronceado y moderno, con gafas de sol. Aprieta la mandíbula, con la camisa abierta de manera un tanto insolente, posando como una estrella mediática.

This is another 'not-the-familiar' Shakespeare [...] A postmodern joke bringing the iconic bald forehead and page-boy hair together with equally iconic local clones, this Dude invites interest but tells interpreters to fuck off (Reynold y West 2005: 3). 


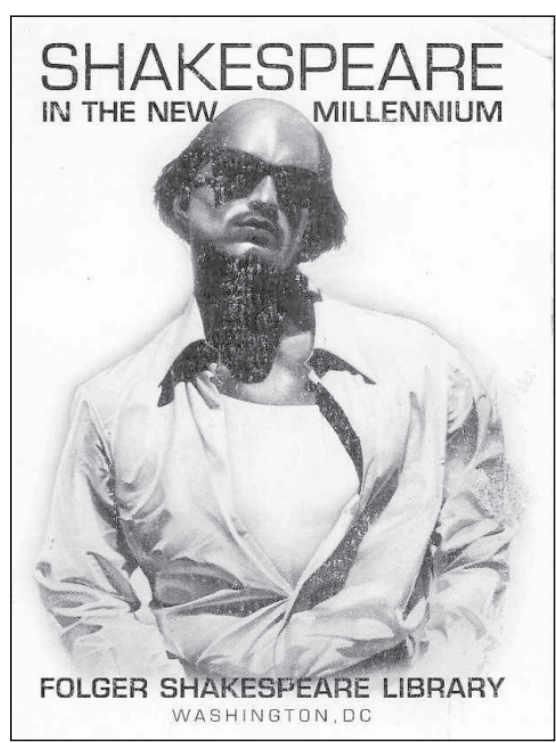

Shakespeare Dude. Kevin Chadwick. Circa 2005. Fuente: Palgrave Macmillan 2005.

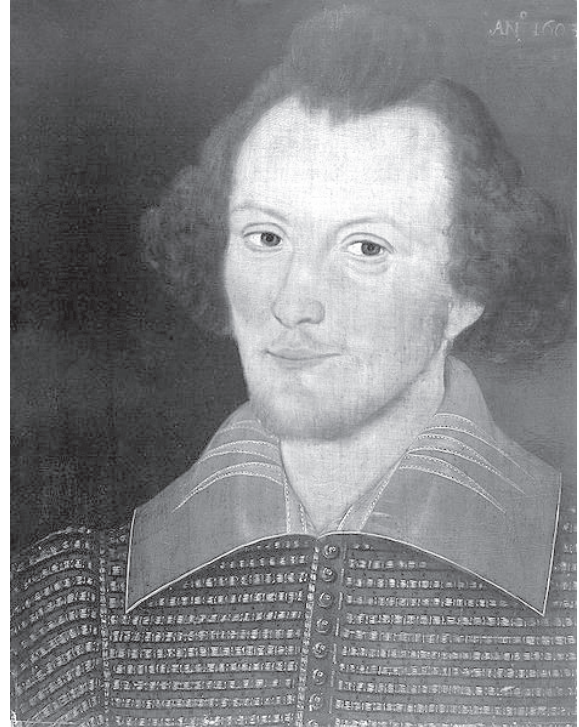

Sanders Portrait. 1603. Fuente: Wikimedia.

En la fecha en la que Reynolds y West publicaron Rematerializing Shakespeare (2005) el Retrato Sanders, había despertado una gran expectación, lo que sugería una suerte de interés generalizado por imaginar a la persona "Shakespeare" como icono cultural" (2005: 9). Es decir, la imagen de Shakespeare ha cobrado una entidad propia que se superpone a sus textos, suscitando en ocasiones incluso mayor interés que los mismos.

De forma similar, el Shakespeare Institute de Stratford-upon-Avon y la Folger Shakespeare Library en Washington han optado por reproducir el retrato de Shakespeare con la estética Pop de las tintas planas y los contornos delimitados heredada de Andy Warhol con el ánimo de publicitar distintos eventos organizados en sus inmediaciones. Más allá de estos ejemplos, una nueva ola de retratos de Shakespeare también ha dejado huella en el arte urbano, como demuestra el graffiti que en 2008 realizó una mano anónima (tal vez Banksy) en la pared de un almacén neoyorquino. 


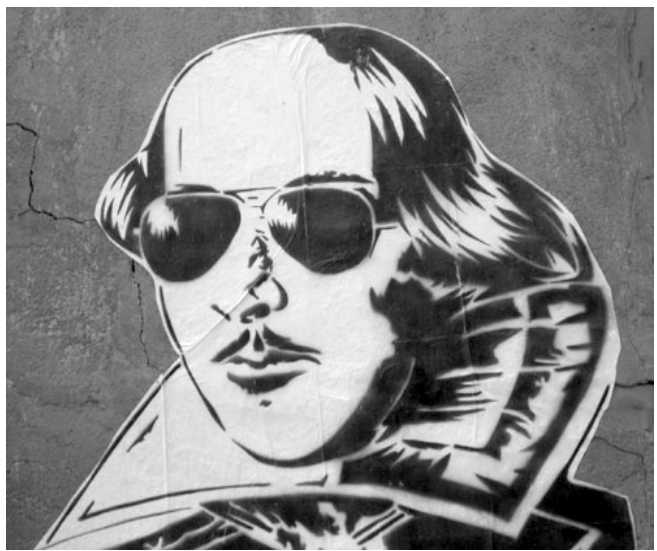

William Shakespeare with Aviator Sunglasses. Graffiti anónimo en Nueva York. 2008. Fuente: Globalgraphica.com. Foto de Ivan Corsa.

Queda patente que, a la vez que persiste un interés generalizado en estudios literarios y en el ámbito artístico por recuperar la figura de Shakespeare, algunos sectores han ido adquiriendo cierta consciencia de la posibilidad de reactivar la imagen de Shakespeare, más allá de cuál sea su "original", sin aspirar, necesariamente, a retratarlo (o leerlo) al pie de la letra. Serigrafiar su retrato -y no necesariamente su retrato oficial, aunque sí uno que resulte reconocible-, colorearlo con spray sobre un muro, ponerle las gafas de sol de moda o vestirlo como una estrella de Hollywood son gestos habituales de "rematerialización":

To rematerialize is not to recover a lost material infrastructure $[\ldots]$ nor is it to restore to some existing material its priority over the imaginary. Indeed [...] the material is incomplete without the supplements of the imagination, the intended and the desired (Reynold y West 2005: 9).

Posiblemente, la imagen más iluminadora en relación a este reciclaje o rematerialización conjunta de Shakespeare, su obra y las lecturas posibles de dicha obra, sea la que nos ofrece Jean-Luc Godard en la película King Lear, criticada y desaparecida nada más estrenarse en 1987, pero reeditada en 2009 al auspicio del creciente interés tanto por la obra más experimental de Godard como por las apropiaciones y ensayos radicales en torno a Shakespeare. En su particular adaptación, Godard bosqueja su propio retrato de Shakespeare al incluir a su descendiente, Shakespeare Junior V (Peter Sellars), entre los personajes principales. A través de él, la rematerialización de Shakespeare tiene lugar a varios niveles. Por un lado, nos hallamos ante el legado 'genético' del bardo, su pervivencia biológica se concentra en la persona de Shakespeare Junior. Si bien éste no es un retrato al pie de la letra de Shakespeare, resultado de la creación de un molde exacto del rostro de su antecesor, es pertinente anotar que subrayar la consaguinidad entre ambos, metafóricamente o no, implica justamente 
relacionarlos de manera directa, legitimando de antemano la autoridad del retrato con respecto al modelo. ${ }^{7}$ Por otro lado, Shakespeare Junior, representante de la compañía Canon, es el encargado de buscar y reconstruir los restos de la cultura occidental, destruida tras el desastre apocalíptico de Chernobyl. Este Shakespeare, apenas consciente del significado de las frases que rescata, bien podría ser ese tipo de artista del que habla Perniola (a partir de Clément Rosset) y que sucede "al artista como genio de Schopenhauer y de la tradición romántica." Se trata del "artista como idiota; lo que tienen en común estas dos figuras, a primera vista antitéticas, es la pretensión de captar la esencia de lo real más allá de todas las mediaciones falaces del lenguaje y del pensamiento" (2002: 25).

Entre sus redescubrimientos, el legado literario de William Shakespeare, justamente, es el que dota de unidad a la película. Rematerializados por las voces de los personajes de la película, los versos de Shakespeare sobrevuelan el montaje visual, hilos de una densa red sonora que Shakespeare Junior escucha atento, anotando, a veces, palabras en su inseparable libreta. Vestido de negro y dirigiendo la mirada al horizonte, en una imagen que hace confluir el recuerdo de Hamlet (de fondo, escuchamos fragmentos del monólogo 'to be or not to be'), el Lear de Kozintsev y el cuadro de Gaspar David Friedrich, El viajero ante un mar de nubes (1818), el joven Shakespeare Junior encarna el punto de encuentro de distintos tiempos históricos. La materia shakesperiana, con los suplementos de la nueva era que la ve renacer, hace presente el pasado derruido, reinventado.
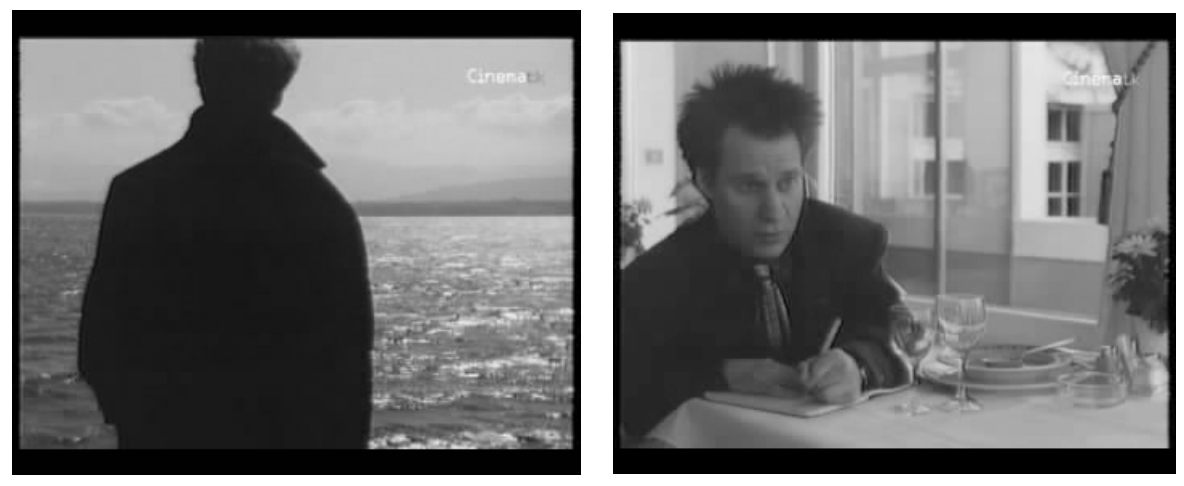

Shakespeare V. Jean-Luc Godard. 1987. Fuente: captura de fotograma de la película King Lear.

En contraste con las perspectivas descritas al principio de esta sección, (recordemos: la búsqueda artística idealizadora de Gainsborough, el rastreo histórico -también idealizadorde Stanley Wells, etc.), donde se relacionaba la autenticidad con una unidad homogénea, concreta, perteneciente a un tiempo pasado absoluto, el Shakespeare que nos propone Jean-

7 Por supuesto, esta relación parental no carece de cierta ironía. La película fuerza hasta sus extremos, hasta parodiarla, la idea de la legitimidad de la adaptación de una obra clásica como King Lear. 
Luc Godard encarna una autenticidad heterogénea. Shakespeare V es producto (y productor) de un fenómeno cultural complejo que no entiende de tiempos absolutos. Reconocemos en él los resquicios de su antepasado, pero éstos no llegan a conformar significados -parece querer decir Godard- si no se tiene en cuenta toda la red de relaciones (de voces) que se superpone a ellos.

No obstante, las dos posturas que aquí he señalado (revival y reciclaje o rematerialización) no son opuestas sino que tienen mucho en común. En los reciclajes que llevan a cabo Godard, Chadwick y el grafitero anónimo reaparece el Shakespeare que reconoce nuestra memoria; yace en ellos un recuerdo a contrapelo del tiempo. No pertenecen a un instante puramente presente. Asimismo, las aspiraciones revivals de artistas como Gainsborough o historiadores como Stephanie Nolen (descubridora del retrato Sanders) participan a su manera en la construcción de una identidad shakesperiana inconstante en el presente. Cada 'descubrimiento' del retrato auténtico, ha ido añadiendo, paradójicamente, un eslabón en la cadena de (hasta el momento) retratos improbados, escribiendo así una curiosa Historia de Falsos Retratos. La labor de reciclaje (y su capacidad, por tanto, para añadir un suplemento -ese símbolo '+', ese carácter extra-shakesperiano) del revival tiene un impacto similar, a veces incluso mayor, en la reinvención y recepción de Shakespeare que la de los artistas como Godard o los teóricos presentistas. La diferencia entre unos y otros, entonces, reside en las premisas de las que parten; básicamente diferentes concepciones de 'tiempo' y 'autenticidad'.

Lo común entre unos y otros es esa capacidad de cambio a partir de la manipulación y montaje de los restos de Shakespeare a la vez que el carácter inamovible, resistente y superviviente de dichos restos -o, como decíamos anteriormente, 'supervivencias' en el sentido warburgiano del término. Esta cualidad liminal nos ofrece, para concluir, una nueva perspectiva sobre la figura de Shakespeare más allá del ideal romántico y del nihilismo postestructuralista. Tal y como han sugerido Marjorie Garber y Courtney Lehmann, podemos considerar a Shakespeare un 'escritor fantasma' (a Ghostwriter); en definitiva, una presencia que se proyecta de diversas formas y de la que participan diversos tiempos, al tiempo que un autor en la sombra, pese a los focos. A partir de él, cada cual escribe su propia historia.

\section{REFERENCES}

Bate, J. 2003. “The Shakespeare Phenomenon.” Eds. Jane Martineau et Al. Shakespeare in Art. London and New York: Merrel. 3-31.

2010. Soul of the Age: A Biography of the Mind of William Shakespeare. New York: Random House Trade Paperbacks.

BARTHES, R. 2009. “La muerte del autor.” El susurro del lenguaje. Barcelona: Paidós.

Baugh, C. 2003. "Our Divine Shakespeare Fitly Illustrated. Staging Shakespeare 1660-1900.” Eds. Jane Martineau ET AL. Shakespeare in Art. London and New York: Merrel. 29-47.

Bloom, H. 1999. The Invention of the Human. New York: Riverhead Trade. 
Bryson, Bill. 2007. Shakespeare: The World as Stage. London: Harper Perennial.

Cartelli, T., Rowe, C. 2007. New Wave Shakespeare on Screen. Malden: Polity Press.

CARTMELL, D. 2000. Interpreting Shakespeare on Screen. London: Palgrave Macmillan.

Clair J. 1999. Elogio de lo visible, Barcelona: Seix Barral.

Courtney L., 2002. Shakespeare Remains: Theater to Film, Early Modern to Postmodern. Ithaca and London: Cornell University Press.

De Grazia, M., Stallybrass, P. 1993. "The Materiality of the Shakespearean Text." Shakespeare Quarterly 44, 3: 255-283.

DunCAn-Jones, K. 20 March 2009. “Unfound(ed)?” Times Literary Supplement.

Godard, J. 1987. King Lear. Cannon. DVD.

Hartman, S. 1901. Shakespeare in Art. Boston: L.C Page and Company.

Howard J., O'Connor, M. 2004. Shakespeare Reproduced: The Text in History and Ideology. New York: Routledge.

JACKSON, R., ed. 2007. The Cambridge Companion to Shakespeare on Film. Cambridge: Cambridge University Press.

KNIGHT, J. 2002. Shakespeare and Religion. London: Routledge.

LaCAYo, R. 2009. "Is this What Shakespeare Looked Like?” Times. US edition. 9 Sept. 2015. http://content.time.com/time/arts/article/0,8599,1883770,00.html.

Lehmann, C. 2000. Shakespeare Remains: Theater to Film, Early Modern to Postmodern. London: Ithaca and London: Cornell University Press.

Orgel, S. 2002. The Authentic Shakespeare. London and New York: Routledge.

Perniola, M. 2002. El arte y su sombra. Madrid: Cátedra.

Reynolds, B., West, W. 2005. Rematerializing Shakespeare. Authority and Representation on the Early Modern English Stage. London: Palgrave Macmillan.

SHAPIRO, J. 2005. A Year in the Life of William Shakespeare: 1599. New York: HarperCollins.

TwaIn, M. 2004. Is Shakespeare Dead? Whitefish, Montana: Kessinger Publishing.

WArburg, A. 1999. "The Renewal of Pagain Antitiquity.” Eds. Julia Bloomfield ET AL. Contributions to Cultural History of the Renaissance in Europe. Los Angeles: Getty Research Institute.

Wells, S. 1995. Shakespeare, a Life in Drama, New York and London: W.W. Norton \& Company.

2003. Shakespeare of All Time. Oxford: Oxford University Press.

2009. "Professor Stanley Wells, Chairman of The Shakespeare Birthplace Trust discussing the Cobbe Portrait." Shakespeare Found. 
Stratford-upon-Avon.Marzo-Octubre2009. http://www.shakespearefound.org.uk/ evidence.html/

Woudhuysen, H. 2009. "The Claims for the Cobbe Portrait. "Shakespeare Found. Stratford-upon-Avon.Marzo-Octubre2009. http://www.shakespearefound.org.uk/ evidence.html/ 
